\title{
A PLL Control for Self-Tuning of Parallel Wireless Power Transfer Receivers Utilizing Switch-Mode Gyrator Emulated Inductors
}

\author{
Mohamed Saad ${ }^{1}$, Herminio Martínez-García ${ }^{1}$, Pedro Alou ${ }^{2}$ and Eduard Alarcón ${ }^{1}$ \\ ${ }^{1}$ Department of Electronics Engineering, Universitat Politècnica de Catalunya, Barcelona, Spain \\ ${ }^{2}$ Centro de Electrónica Industrial - CEI, Universidad Politécnica de Madrid, Madrid, Spain \\ C/Jordi Girona, 1-3, Edificio C4, 08034 \\ Barcelona, Spain \\ E-Mail:m.saad@eng.aswu.edu.eg, eduard.alarcon@upc.edu
}

\section{Acknowledgements}

This work has been partially funded by the Spanish Ministry of Science and Innovation (projects DPI2013-47799-C2-1-R and DPI2013-47799-C2-2-R).

The first author would like to thank the Egyptian Ministry of Higher Education for his $\mathrm{PhD}$ grant to study in UPC BarcelonaTech.

\section{Keywords}

«Contactless Energy Transfer», « Switch-Mode Gyrator», «Active Inductor», «Self-Tuning», «Wireless Power Transfer»

\begin{abstract}
In multiple receivers wireless power transfer (WPT) systems, it is preferable to retune the resonant frequency of every receiver to the transmitter operating frequency in front of frequency mismatches. This paper discusses a proposal for electronic tuning for WPT receivers by means of a variable active switch-mode inductance. The proposed method benefits from the gyrator concept to emulate a variable inductance. Instead of the conventional approach of linear amplifier based implementation of a gyrator, a switch-mode gyrator circuit is exploited for more efficient operation. Additionally, a PLL-like control is presented to enable self-tuning for the receiver resonant tank. Furthermore, a design-space characterization for the system dynamic behavior has been discussed to show the control robustness and the instabilities (including slow-scale and fast-scale chaotic instabilities) it may undergo.
\end{abstract}

\section{Introduction}

Resonant Inductive Coupling Wireless Power Transfer (RIC-WPT) has become a topic of wide interest in research and industry in the last two decades. A reasonable number of applications has been enabled by RIC-WPT, whenever the necessity for a contactless power transmission arises.

RIC-WPT is characterized by resonating the transmitter and receiver at a particular frequency [1]. Considering high-Q transmitter and receiver, even a small mismatch in the resonant frequency of RICWPT's receiver gives rise to a significant drop in the received power [2]. Possible mismatch origins include, but are not limited to, component tolerance, distance variations and interference from close proximity devices. In real life, such sources of mismatch are random, unpredicted and mostly inaccessible [3].

RIC-WPT has been considered as a key enabling technology for multiple receiver systems where a single transmitter is cross-coupled to multiple receivers [4]. Unlikely, assuming an identical mistuning percentage at each receiver is impractical in a very dynamic environment, especially in the case of crosscoupling with other receivers [3],[5]. Therefore, it is not feasible to tune the operating frequency at the 
transmitter side. Matching the resonant frequency of each receiver autonomously to a single operating frequency is more straightforward and flexible approach [3].

Different solutions have been proposed in literature for a seamless tuning of RIC-WPT receivers. In [6], a switched capacitor matrix has been proposed as an adaptive impedance matching for compensating the effects of distance change. Another approach is to add a saturable-core inductor in parallel with the receiver resonant circuit where the resonant frequency could by continuously tuned [5]. However, such approach is not advantageous for smaller devices in terms of occupied size. Adding a dynamic switched inductor in parallel with the receiver circuit is another approach where the effective inductance or capacitance of the receiver circuit can be varied, hence, the resonance frequency can be tuned [7], [8]. On the other hand, an LCL receiver circuit can be used where the resonant circuit is tuned by including a controller LCL actuator [9], or a magnetic amplifier [10]. A further approach of using DC-DC converters for active tuning has been introduced in [11], where a DC-DC converter is modified to inject a negative or positive reactance which could be used to control the receiver resonant frequency.

In this paper, an idea of tuning parallel-compensated RIC-WPT receivers using a variable active inductance is proposed. The variable active inductance is realized by means of a switch-mode gyrator circuit. In addition, a phase locked loop (PLL)-like control method is introduced which enable selftunability for the receiver resonant circuit.

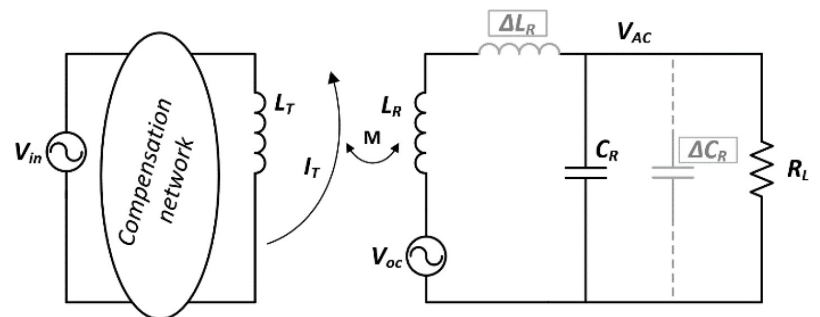

Fig. 1: Simplified model for WPT parallel receiver

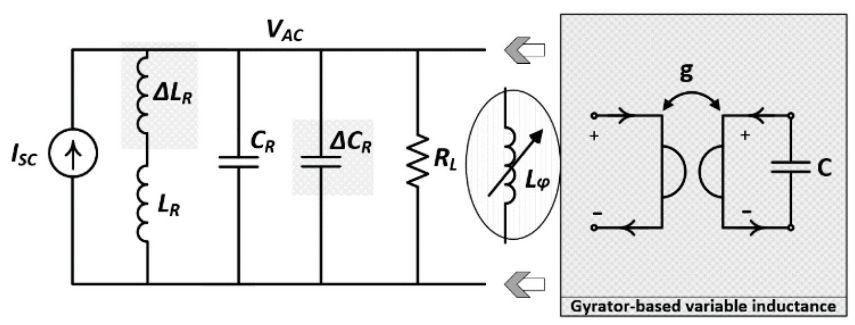

Fig. 2: Gyrator-based variable $L$ for receiver tuning

\section{Principles of Parallel-Compensated RIC-WPT Receiver}

In parallel-compensated RIC-WPT receivers, a capacitor is connected in parallel with the receiver coil to enable the receiver to resonate precisely to the transmitter frequency $f_{T}$. Considering the simplified model given in Fig. 1, the resonant tank output voltage $V_{A C}$ can be given by:

$$
V_{A C}=\frac{V_{o c}}{\left(1-\omega^{2} L_{R} C_{R}\right)+j \omega L_{R} / R_{L}}
$$

where $V_{o c}$ is given as:

$$
V_{o c}=j \omega M I_{T}
$$

where $M$ is the mutual inductance between the transmitter and receiver is coils and $I_{T}$ is the transmitter coil current. Under fully-tuned resonant condition (i.e., $\omega_{R}=1 / \sqrt{L_{R} C_{R}}$ ), then (1) reduces to:

$$
V_{A C}=-j \frac{R_{L}}{\omega L_{R}} V_{o c}=-j Q_{R L} V_{o c}
$$

where $Q_{R L}$ is the quality factor of the receiver resonant circuit. The quality factor $Q_{R L}$ is the voltage boosting factor as implied by (3), where $Q_{R L}$ is merely load-dependent factor assuming lossless reactive components.

The power transmitted to the receiver can be derived from the real part of the reflected impedance from the receiver to the transmitter. The reflected impedance to the transmitter is given by:

$$
Z_{\text {ref }}=\frac{\omega^{2} M^{2}}{Z_{R}}=R_{\text {ref }}-j X_{\text {ref }}
$$


where $Z_{R}$ is the receiver input impedance, and $R_{\text {ref }}, X_{\text {ref }}$ are the real and imaginary parts of the reflected impedance respectively. The expressions for $R_{r e f}$ and $X_{r e f}$ are as follows:

$$
\begin{gathered}
R_{\text {ref }}=\frac{\omega^{2} M^{2} R_{L}}{\left(\omega^{2} L_{R} C_{R} R_{L}-R_{L}\right)^{2}+\left(\omega L_{R}\right)^{2}} \\
X_{\text {ref }}=\frac{\omega^{3} M^{2}\left[C_{R} R_{L}^{2}\left(\omega^{2} L_{R} C_{R}-1\right)+L_{R}\right]}{\left(\omega^{2} L_{R} C_{R} R_{L}-R_{L}\right)^{2}+\left(\omega L_{R}\right)^{2}}
\end{gathered}
$$

When the receiver resonant circuit is fully-tuned, then (5) reduces to:

$$
R_{\text {ref }}=\frac{M^{2} R_{L}}{L_{R}^{2}}=\frac{\omega_{o} M^{2} Q_{R L}}{L_{R}}
$$

The power transmitted to the receiver is the power dissipated into the real part of the reflected impedance, which reaches a maximum at fully-tuned receiver. The maximum RMS power transmitted can be shown as [5]:

$$
P_{\max }=\frac{\omega_{o} Q_{R L} M^{2} I_{T}^{2}}{L_{R}}
$$

The maximum power in (8) refer to the load dependency, moreover, power transfer capability is greatly enhanced with higher quality factor $Q_{R L}$. However, the receiver circuit becomes more sensitive for any variations as $Q_{R L}$ increases. Fig. 1 depicts possible variations in $L_{R}$ or $C_{R}$ modeled as $\Delta L_{R}$ and $\Delta C_{R}$ respectively, where $\Delta L_{R}$ and $\Delta C_{R}$ can be in positive or negative values. Including such variations in (1) or (5), yields a shift in the resonant frequency of the receiver. Consequently, a significant drop in the received power is inevitable and (8) is no longer applicable. In order to retune the resonant frequency of the receiver, an adaptive tunable element can be used to nullify the effect of component variations.

\section{Proposal of Parallel Tuning system for RIC-WPT Receivers}

In order to counteract the possible mistuning in RIC-WPT receivers, the resonance frequency of the receiver circuit can be re-tuned by means of an additional correcting inductive or capacitive element. Obviously, the added element has to be an active element where the feature of tunability is met. Fig. 2 shows a simple model for the WPT receiver with the connection of a variable emulated inductance. The emulated inductance is based on the concept of gyrator-C.

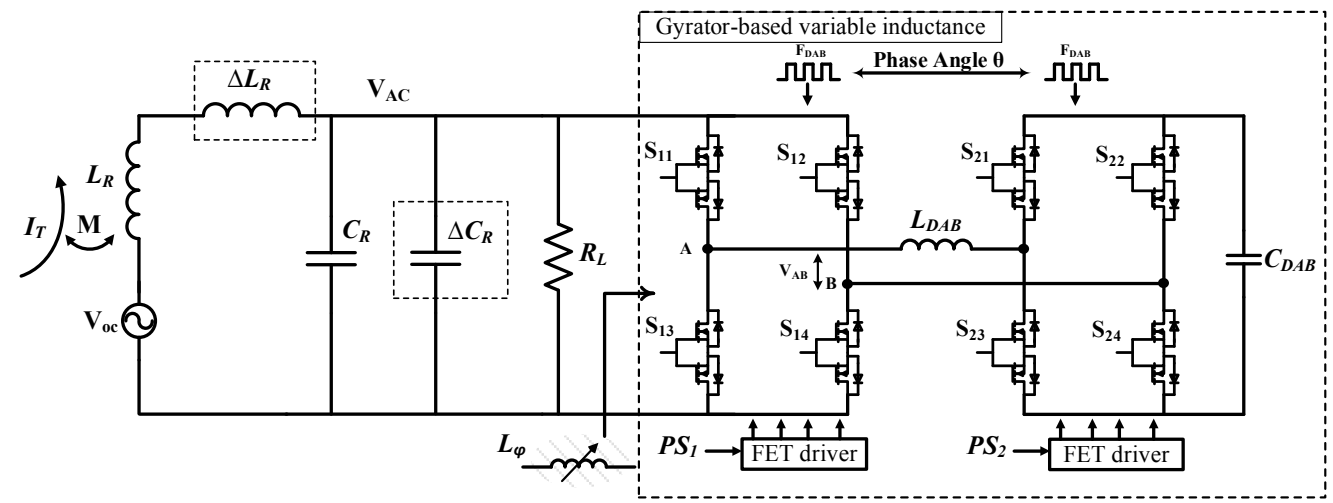

Fig. 3: The proposed self-tuning of WPT parallel receivers including schematic diagram of a gyrator.

The emulated inductance $\left(L_{\varphi}\right)$ is connected in parallel with the compensation capacitor. Therefore, it can be deemed as an additional inductance connected in parallel with the receiver coil $L_{R}$ (and $\Delta L_{R}$ in essence) by examining the Norton equivalent of receiver circuit in Fig. 2. Therefrom, the resonance frequency of the receiver circuit after adding $L_{\varphi}$ can be given as:

$$
\omega_{R}=\frac{1}{\sqrt{L_{R} C_{e}}}
$$


where $C_{e}$ is the equivalent compensation capacitance after the application of variable emulated inductance $\left(L_{\varphi}\right)$ :

$$
C_{e}=C_{R}-\frac{1}{\omega^{2} L_{\varphi}}
$$

It is implied from (9) that the resonance frequency can be tuned appropriately over a wide range that is merely limited by $L_{\varphi}$ tuning range. The added variable term in (10), i.e. $\frac{1}{\omega^{2} L_{\varphi}}$, can be employed to eliminate the effect of any variations in the receiver resonant circuit, e.g. $\Delta L_{R}$ and $\Delta C_{R}$.

Fig. 3 shows a diagram for the gyrator circuit that is proposed for realization of emulated inductance. The operation of the gyrator circuit is described in the next section.

\section{DAB Gyrator-Enabled Variable Inductance}

Gyrator is classified as a lossless, two-port, linear and power-conservative network element. As a consequence of its non-reciprocity feature, a gyrator is able to emulate an inductive reactance at one port while a capacitance is being connected to the other port. This feature has always been of wide interest for signal-processing circuits to replace bulky inductors by on-chip integration of a gyrator-C circuits [12], in which a gyrator is synthesized by means of linear amplifiers. Unlikely, conventional gyrator implementations using linear amplifier is not considered as a potential candidate for power applications due to poor efficiency. Consequently, a loss-free synthesis for gyrator is essential for power processing application [13].

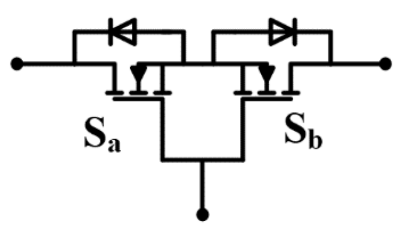

Fig. 4: Four-quadrant bidirectional switch

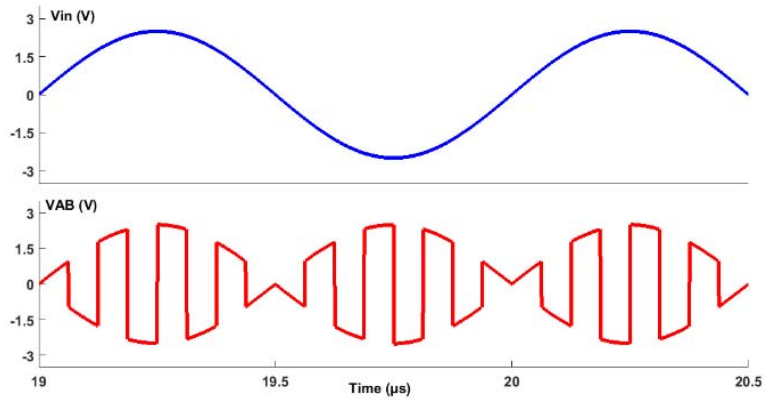

Fig. 5: Bridge output voltage in response to ac signals

A topology of wide interest for many power-processing applications is the Dual active bridge (DAB) converter. The DAB has been validated as a switch-mode natural gyrator without the need to force the gyration functionality via a devoted control [14]. The operation and application of DAB converter for inductance emulation has been discussed, in details, in [15], [16]. The schematic diagram of the DAB circuit is shown in Fig. 3, where the connection to the WPT receiver is indicated as well. The DAB converter is composed of two full-bridge $\left(S_{11}-S_{12}-S_{13}-S_{14}\right.$ bridge and $S_{21}-S_{22}-S_{23}-S_{24}$ bridge in Fig. 3) linked by an AC inductor. Moreover, it is a bidirectional topology where the power flow direction is guided by the relative phase-shift $(\varphi)$ between the two bridges. The DAB converter has been primarily as a DC-DC converter for the purpose of power processing where all the converter switches shown in Fig. 3 could be realized by a two-quadrant IGBT or FET switch. However, the synthesis of variable reactive components using the same converter imposes the capability of processing ac signals either at input or output sides. Thus, the DAB converter operation must allow bidirectional current flow and voltage polarity [17]. Then all the switches in Fig. 3 have to be realized by four-quadrant switch, e.g. the two back-to-back switch as the MOSFET type shown in Fig. 4. As a result, a bipolar square-wave can be produced at the first bridge output $V_{A B}$ in response to a sinusoidal input as shown in Fig. 5.

If a capacitor $C_{D A B}$ is connected to the output of the $\mathrm{DAB}$, then the equivalent inductance can be formulated as [16]:

$$
L_{\varphi}=\frac{C_{D A B}}{\mathrm{~g}^{2}}=\frac{4 \pi^{4} f_{D A B}^{2} L_{D A B}^{2}}{\left(\varphi \pi-\varphi^{2}\right)^{2}} \cdot C_{D A B}
$$

where $\mathrm{g}$ is the gyration ratio of the $\mathrm{DAB}, f_{D A B}$ is the switching frequency of the $\mathrm{DAB}$ converter, and $L_{D A B}$ is the link inductor. Implied by (11), the emulated inductance $L_{\varphi}$ can be varied and tuned by tuning 
the phase-shift value $\varphi$. To highlight the gyration behavior of DAB circuit, Fig. 6 shows how the gyration ratio, as well as, the emulated inductance comply with a phase-shift sweep from $30^{\circ}$ to $150^{\circ}$, where the DAB specifications for this simulation setup are $\left(f_{D A B}=8 \mathrm{MHz}, L_{D A B}=25 \mathrm{nH}, C_{D A B}=\right.$ $80 \mathrm{nF}$ ). One important conclusion from Fig. 6 is that $\mathrm{g}$, and thus $L_{\varphi}$, shows a symmetric response around $\left(\varphi=90^{\circ}\right)$. Consequently, variation range of the emulated inductance is limited by phase-shift range of $\left(\varphi \cong 0^{\circ} \rightarrow \varphi \cong 90^{\circ}\right)$ or $\left(\varphi \cong 90^{\circ} \rightarrow \varphi \cong 180^{\circ}\right)$. The $\mathrm{DAB}$ converter is based on the concept of AC inductor which means that the average current in $L_{D A B}$ is zero. The input current of the DAB converter is therefore discontinuous. Fig. 7 shows simulation results for a DAB converter chopping a $1 \mathrm{MHz}$ sinusoidal input with a rate of $8 \mathrm{MHz}$. It is valid to infer the DAB input current by handling the DAB output as an indication, a fact that is derived from the gyrator characteristic equation $\left(i_{i n}=\mathrm{g} \cdot v_{o}\right)$. Thus, Fig. 7 manifest the quadrature phase-shift between $v_{i n}$ and $v_{o}$ of the DAB as an evident for inductive behavior at the converter input.

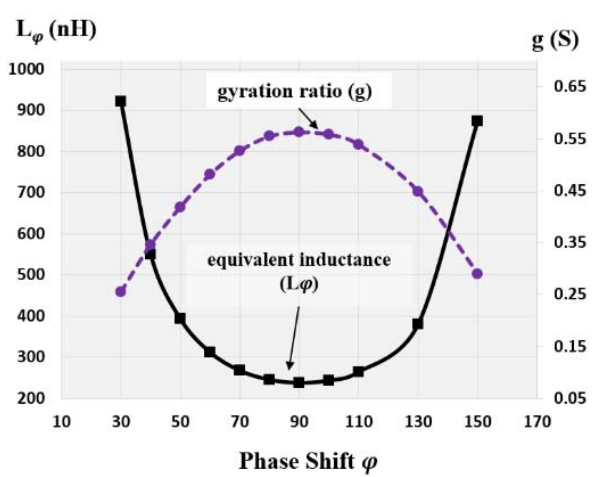

Fig. 6: g and $L_{\varphi}$ vs phase-shift $\varphi$

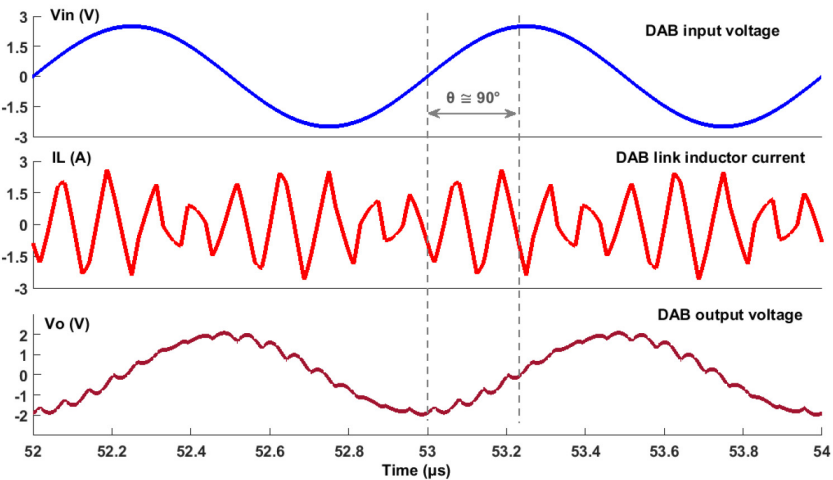

Fig. 7: Bridge output voltage in response to ac signals

\section{A PLL Control Design}

\section{The proposed Control}

In order to enable self tunability for the proposed system, a controller is required to ensure that the WPT receiver is fully tuned in response to any variation in circuit parameters. The objective of the controller is to check whether there is a mistuning in the receiver tank, then it acts by fine-tuning the phase-shift $\varphi$ of the DAB converter that corresponds to a specific $L_{\varphi}$ which is necessary for retuning. The proposed control is based on the fact that, in a parallel WPT receiver, the resonant tank voltage $V_{A C}$ always lags the coil induced voltage $V_{o c}$ by $90^{\circ}$ while operating at full resonance [11]. The output voltage transfer function of the parallel receiver tuning circuit after adding the DAB variable inductance $L_{\varphi}$ can be given by:

$$
\frac{V_{A C}}{V_{o c}}=\frac{-R_{L} L_{\varphi}}{\omega^{2} C_{R} L_{\varphi} L_{R} R_{L}-\left(L_{\varphi}+L_{R}\right)-j \omega L_{\varphi} L_{R}}
$$

Regard to our proposal, it is of great interest to examine the phase angle between $V_{A C}$ and $V_{o c}$ by converting (12) to the polar form. The phase angle can be expressed as:

$$
\tan \theta=\frac{\omega L_{\varphi} L_{R}}{\omega^{2} C_{R} L_{\varphi} L_{R} R_{L}-\left(L_{\varphi}+L_{R}\right)}
$$

where $\theta$ is the phase angle between $V_{A C}$ and $V_{o c}$. For $\theta$ to be equal to $90^{\circ}$, the right-hand term of (13) has to approach infinity. In other words, the denominator of (13) becomes:

$$
\omega^{2} C_{R} L_{\varphi} L_{R} R_{L}-\left(L_{\varphi}+L_{R}\right)=0
$$

In essence, the controller is a PLL-like approach where a phase detector (PD) and low-pass filter (LPF) are considered for monitoring deviations from resonance state, and hence to close the loop for tracking the fully-tuned condition. The controller block diagram is depicted in Fig. 8. The controller can be envisioned as a PLL-like section, composed of phase detector and low-pass filter, followed by an error amplifier (EA), a PI compensator, and finally a phase-shift modulation (PSM) section. 
To verify the control functionality, the system in Fig. 3 has been used where the DAB gyrator has been used as a variable inductance emulator. The system specifications of the receiver parameters, the controller, and the DAB gyrator are all shown in Table I. As previously stated, the variation of $L_{\varphi}$ is limited between a maximum and minimum values which are determined by DAB phase-shift limitation as $\varphi \cong 0^{\circ}$ and $\varphi=90^{\circ}$ respectively. The DAB parameters, given in Table I., are designed such that the value of $L_{\varphi}$ is used as a part of the resonant tank even at nominal condition, e.g., the compensation capacitor $C_{R}$ is designed to resonate with the combination of $L_{\varphi}\left(\right.$ at $\left.\varphi \cong 45^{\circ}\right)$ and $L_{R}$. The DAB phaseshift $\varphi$ value of $45^{\circ}$ has been selected to enable a wide range bidirectional tuning, i.e. ( $\varphi \cong 45^{\circ} \rightarrow \varphi \cong$ $\left.0^{\circ}\right)$ and $\left(\varphi \cong 45^{\circ} \rightarrow \varphi \cong 90^{\circ}\right)$.

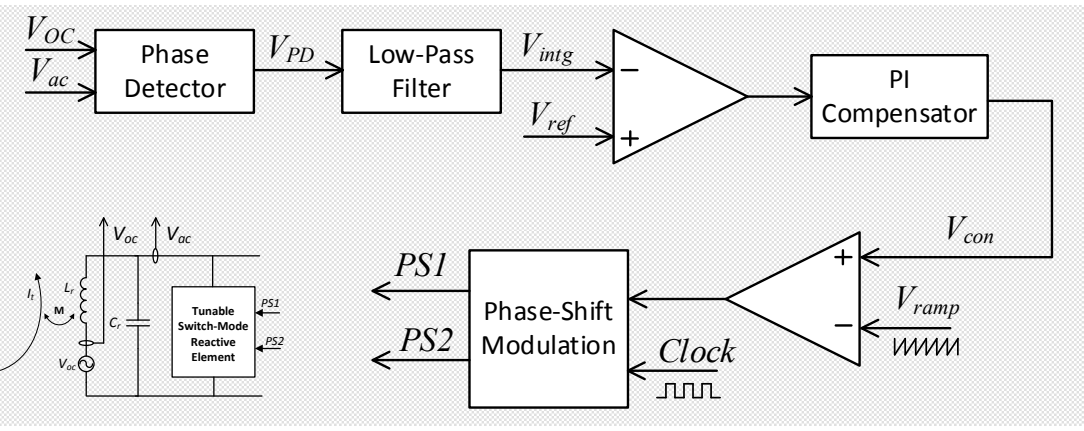

Table I: System parameters

\begin{tabular}{cc}
\hline \hline Parameter & Value \\
\hline$f_{T}$ & $300 \mathrm{KHz}$ \\
$f_{D A B}$ & $5 \mathrm{MHz}$ \\
$C_{D A B}$ & $100 \mathrm{nF}$ \\
$L_{D A B}$ & $66 \mathrm{nH}$ \\
$C_{R}$ & $260 \mathrm{nF}$ \\
$L_{R}$ & $5 \mu \mathrm{H}$ \\
$Q_{R}$ & 15 \\
$f_{L P F}$ & $14.5 \mathrm{kHz}$ \\
\hline \hline
\end{tabular}

Fig. 8: Block diagram of the proposed PLL-like control

\section{Control Validation}

One of the most important steps toward designing a stable controller is to properly design the PI compensator parameters, i.e. the proportional gain $k_{p}$ and the zero frequency $f_{z}$. The system parameters shown in Table I. have been considered as a starting point for validating the control circuit. To obtain a better transient response and faster system dynamics, $f_{z}$ has to be designed for the highest possible value without compromising the stability. However, as seen in Table I., the system involves multiple frequencies (and hence interplay between system dynamics) in the sense that our system is a nonlinear time-varying one. In this case, it is evident that the system bandwidth will not merely be limited by the DAB switching frequency $f_{D A B}$, but the transmitter operating frequency $f_{T}$ as well. The gain $k_{p}$ and zero frequency $f_{z}$ have been designed appropriately such that the aim of a small steady-state error and stable operation is maintained. The full system operation has been verified by simulation where the results are shown in Fig. 9 for $k_{p}=1.5$ and $f_{z}=66.7 \mathrm{kHz}$. On the other hand, an external disturbance has been applied by applying a sudden $+10 \%$ change in $C_{R}$, followed by a $+20 \%$ change in $L_{R}$, which can be shown in Fig. 10.

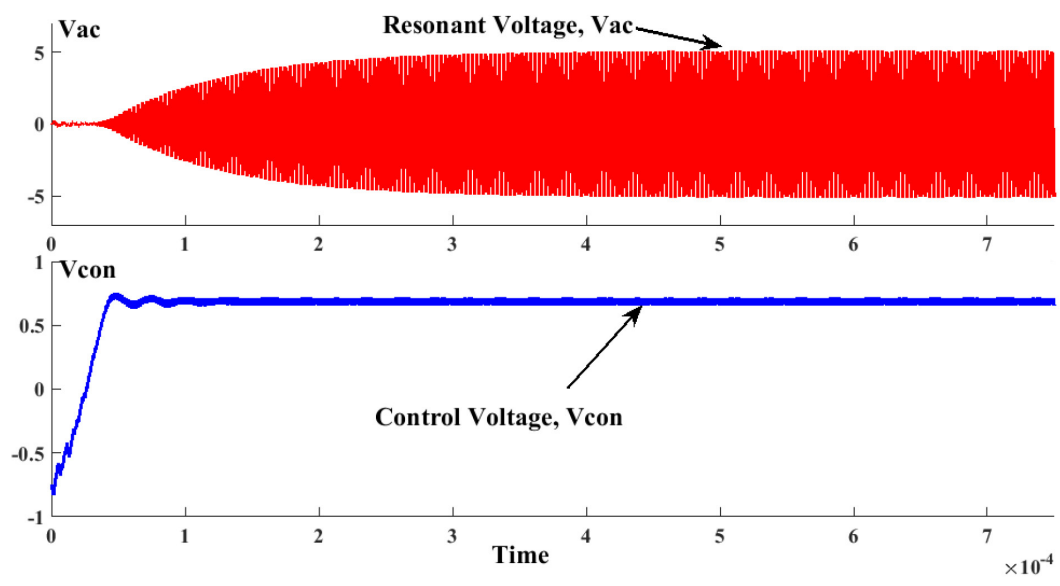

(a) 


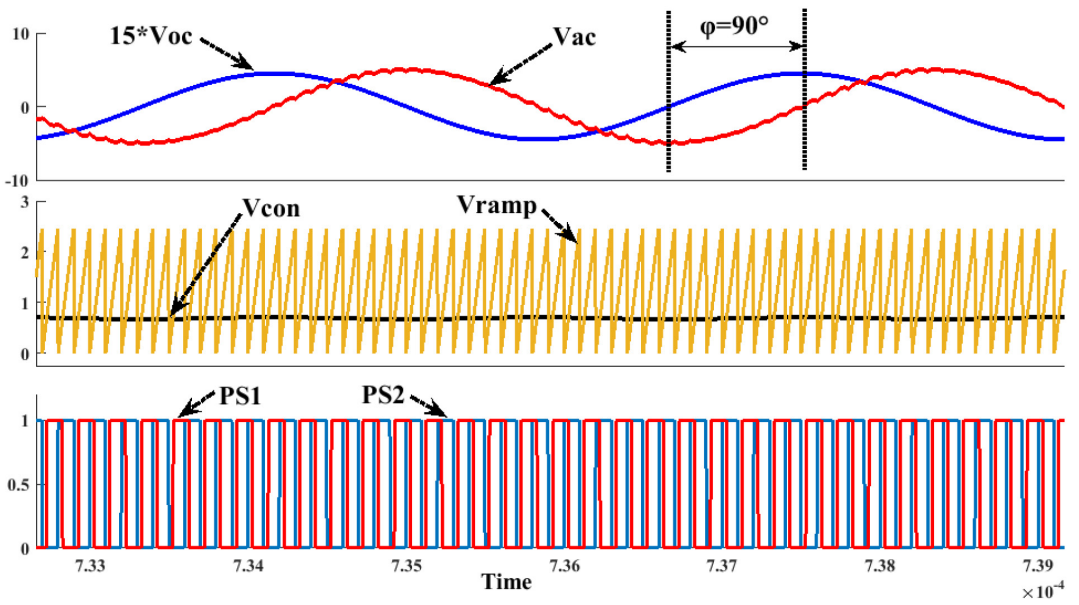

(b)

Fig. 9: System results (a) steady-state results of $V_{\text {con }}$ and $V_{A C}$, (b) a zoom-in showing $15 \cdot V_{\text {in }}, V_{A C}$, $V_{\text {ramp }}, V_{\text {con }}$, and DAB gating signals

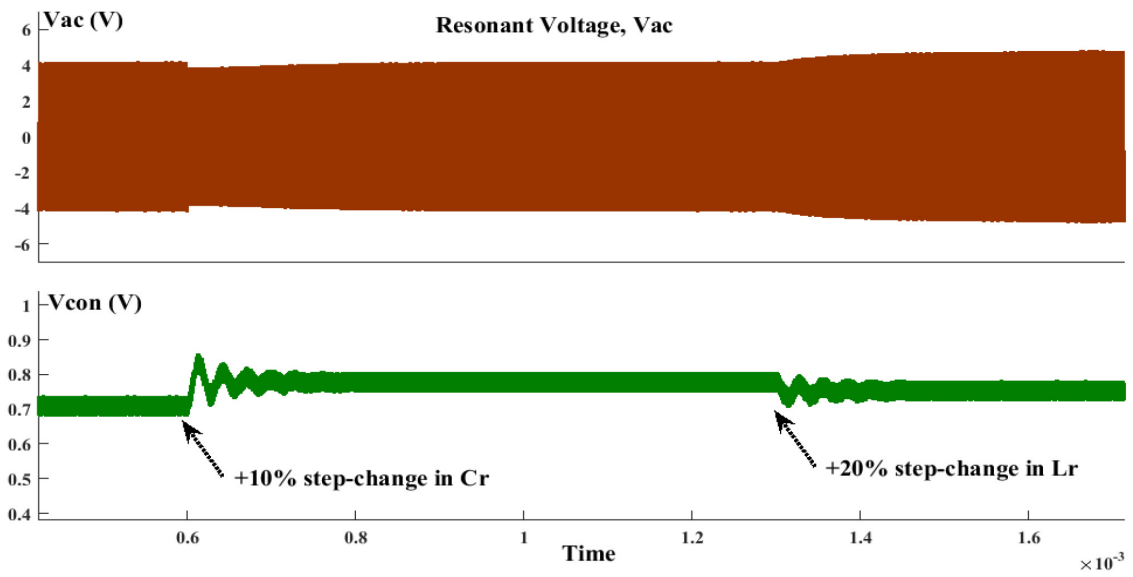

Fig. 10: Control response to variations of $+10 \%$ in $C_{R}$ and $+20 \%$ in $L_{R}$

\section{System Design Consideration}

\section{Efficiency and Quality Factor}

The analysis that has been represented in (1)-(8) has been carried presuming lossless circuit components. In practice, losses in circuit component such as coil windings are inevitable. Furthermore, the gyrator implementation by means of switch-mode DAB converter is meant to be lossless realization. However, the losses associated with the converter switches in the form of switching and conduction losses have a considerable effect on the total quality factor of the receiver circuit. The receiver quality factor $Q_{R}$ given in (7) is no longer applicable once we include the losses of the receiver coil $L_{R}$ and the DAB losses. Fig. 11(a) shows the receiver circuit including coil resistance $R_{R}$ and DAB converter losses as $R_{\varphi}$, while Fig. 11(b) shows the Norton equivalent that used to simplify the analysis. Similarly, the receiver output voltage at resonance can be given as:

$$
V_{A C}=-j Q_{R L} \frac{1}{\left(1+R_{L} / R_{R p}+R_{L} / R_{\varphi p}\right)} V_{o c}=-j Q_{T} V_{o c}
$$

where $R_{R p}$ and $R_{\varphi p}$ are the equivalent values of $R_{R}$ and $R_{\varphi}$ after converting the series network $\left(L_{R}+\right.$ $\left.R_{R}\right)$ and $\left(L_{\varphi}+R_{\varphi}\right)$ to a parallel network. The parallel equivalent $R_{R p}$ and $R_{\varphi p}$ can be given as:

$$
\begin{aligned}
& R_{\varphi p}=\left(1+Q_{\varphi}^{2}\right) R_{\varphi} \cong Q_{\varphi}^{2} R_{\varphi} \\
& R_{R p}=\left(1+Q_{R}^{2}\right) R_{R} \cong Q_{R}^{2} R_{R}
\end{aligned}
$$


where $Q_{R}$ and $Q_{\varphi}$ are the intrinsic quality factors of inductors $L_{R}$ and $L_{\varphi}$ respectively. The circuit losses have a significant effect on the circuit total quality factor $Q_{T}$, a fact that is promoted by (15). It worth to mention that the losses of the DAB has been represented as a series resistance $R_{\varphi}$ while indeed it includes the total energy loss due to switching and conduction loss simultaneously.

In order to maintain a very high $Q_{T}$, the $\mathrm{DAB}$ converter parameters have to be optimized for high efficiency operation. However, deriving equations for power loss in the DAB converter is not straightforward as the system is complex in nature. The power consumed by the DAB converter is a function of switching frequency $f_{D A B}$ which in turn has to be higher than the operating frequency $f_{T}$. In the same time, the losses are closely dependent on the tank output voltage $V_{A C}$ which in turn is a linear function of $Q_{T}$. Consequently, a mathematical modelling for the total efficiency has to be extended while considering the interaction between the $\mathrm{DAB}$ parameters and those of the receiver resonant circuit.

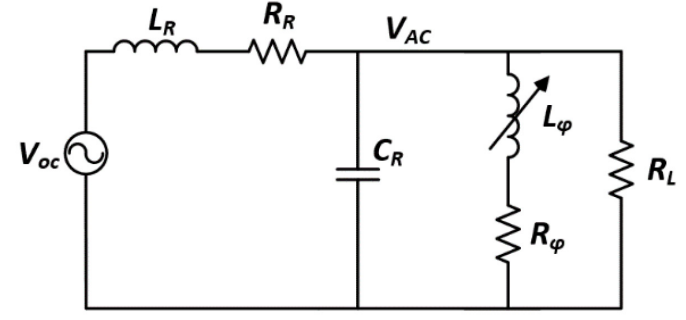

(a)

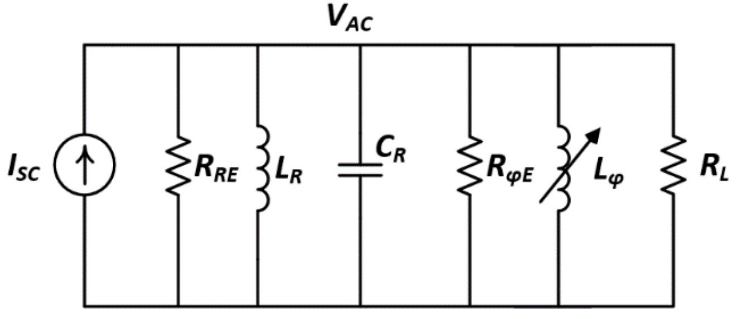

(b)

Fig. 11: Receiver circuit (a) after including inductor losses, (b) Norton equivalent of circuit in (a)

\section{Toward a Design-Oriented Characterization}

In the proposed PLL-like control, a PI control has been used to guarantee a stable tuning operation. However, the system involves multiple correlated parameters, namely, PI zero frequency $f_{z}$, receiver input frequency $f_{T}$, and PWM switching frequency $f_{P W M}$, LPF crossover frequency $f_{L P F}, V_{r e f}, L_{R}, C_{R}$, $C_{D A B}, L_{D A B}$, PWM ramp amplitude $V_{m}$, all which affect the quantitative interplay between the different dynamics of the system. Consequently, the system can exhibit different kinds of instabilities such as slow-scale instabilities (SSI), fast-scale instabilities (FSI) -associated to period-doubling, bifurcation and chaotic regimes [18]. Such kinds of instabilities have been found and characterized for our proposed control. A design-oriented characterization of the system dynamics requires obtaining transient performance under different small- or large-signal excitation for representative cases of the parameter space. Fig. 12 shows the system response where $K_{p}=1.5$ and $f_{z}$ has been shifted to $142.8 \mathrm{kHz}$. It is clear that once $f_{z}$ has been pushed toward the tank operation frequency $f_{T}$, the system has moved from period1 stability region toward SSI region. Moreover, the system tends to show a FSI instabilities for high $K_{p}$ values such as that shown in Fig. 13 at $K_{p}=5.5$ and $f_{z}=66.7 \mathrm{kHz}$.

It is thus possible to design the system at a stable point, however such design may shift due to plant and controller parameter deviations and take different dynamic to other instability regions. The possible routes from a stable starting point can be defined provided that the initial and final states of the system are well defined [18]. Characterizing the full dynamic behavior of the system yet requires an accurate exploration for all the system parameters which yields a more complex but comprehensive bifurcation maps. To limit the scope of this study, a one-dimensional design space for our system has been characterized by performing a parametric sweep over a space of $\left(K_{p}, f_{z}\right)$ as shown in Fig. 14. As depicted, starting from our target design period-1 point of $(1.6,66.7 \mathrm{kHz})$, the system my take different dynamic behaviors that end up in either SSI or FSI regions. 


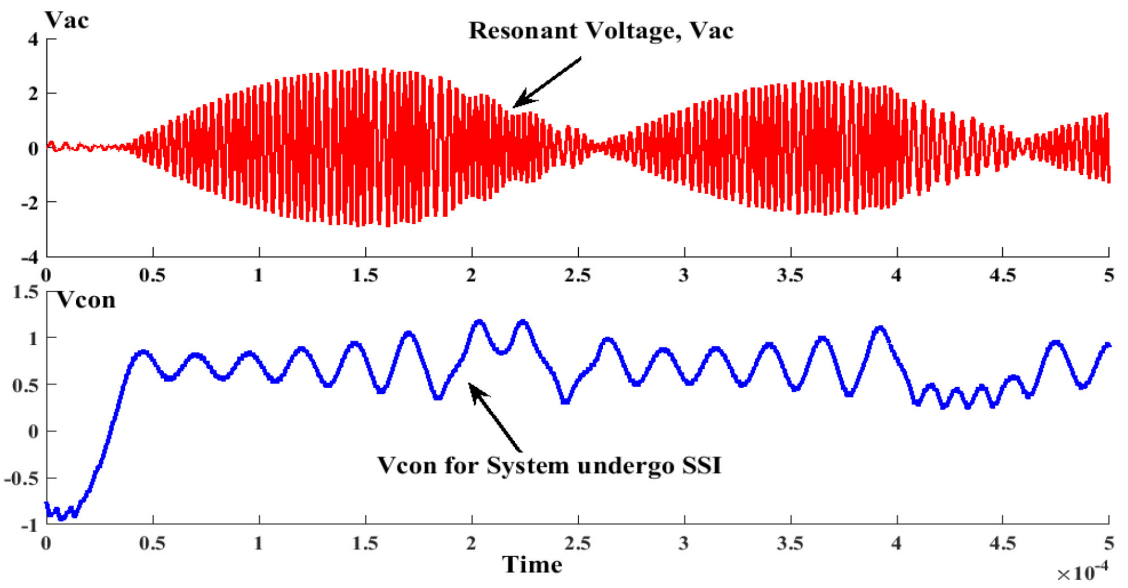

Fig. 12: System SSI at $K_{p}=1.5$ and $f_{z}=142.8 \mathrm{kHz}$

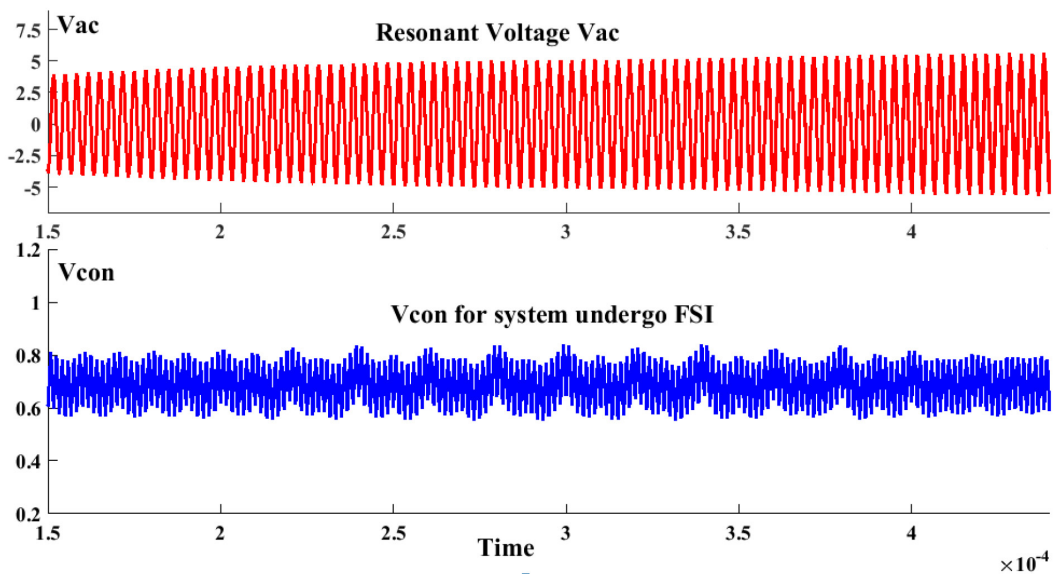

Fig. 13: System FSI at $K_{p}=5.5$ and $f_{z}=66.7 \mathrm{kHz}$

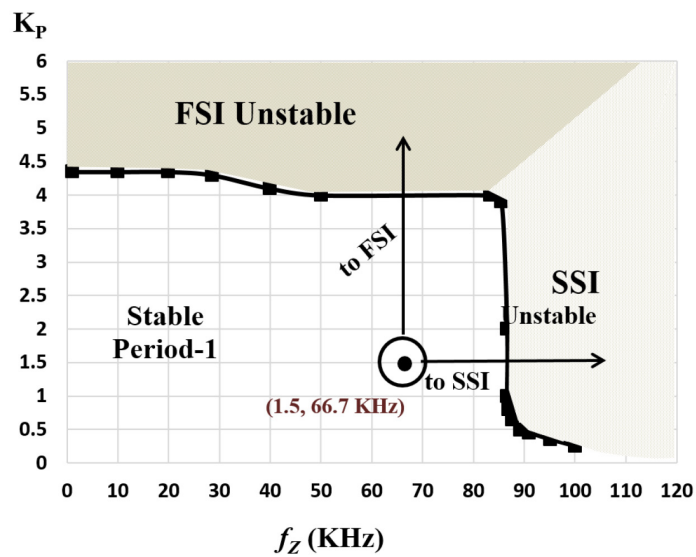

Fig. 14: Characterization of stability boundaries over the space $\left(K_{p}, f_{z}\right)$

\section{Conclusion}

A PLL self-tuning control has been developed and presented for tuning parallel compensated WPT receivers. The proposed technique develops a variable active inductance emulation by means of DAB gyrators. The analysis presented proves that the proposed approach is useful in tuning parallel receivers to retain the maximum power capability of the WPT receiver against any variation in distance or circuit parameters.

Based on this approach, a high switching frequency DAB converter has been connected in parallel to the receiver circuit. The $\mathrm{DAB}$ converter is meant to emulate a lossless variable active inductance that 
has been controlled by a PLL control to guarantee a fully-tuned state in the receiver resonant circuit regardless of any environmental or parametric variations. Moreover, a discussion has been included to address the efficiency and control challenges of the approach. The discussion concludes that the proposed approach is promising for autonomous tuning in WPT receivers provided that a detailed model for the $\mathrm{DAB}$ converter and the whole system is provided.

\section{References}

[1] B. L. Cannon, J. F. Hoburg, D. D. Stancil, and S. C. Goldstein, "Magnetic Resonant Coupling As a Potential Means for Wireless Power Transfer to Multiple Small Receivers," IEEE Trans. Power Electron., vol. 24, no. 7, pp. 1819-1825, Jul. 2009.

[2] X. Mou and H. Sun, "Wireless power transfer: Survey and roadmap," in IEEE Vehicular Technology Conference, 2015, pp. 1-5.

[3] E. Waffenschmidt, "Dynamic Resonant Matching Method for a Wireless Power Transmission Receiver," IEEE Trans. Power Electron., vol. 30, no. 11, pp. 6070-6077, Nov. 2015.

[4] E. Bou-Balust, A. P. Hu, and E. Alarcon, "Scalability Analysis of SIMO Non-Radiative Resonant Wireless Power Transfer Systems Based on Circuit Models," IEEE Trans. Circuits Syst. I Regul. Pap., vol. 62, no. 10, pp. 2574-2583, Oct. 2015 .

[5] M. Zaheer, N. Patel, and A. P. Hu, "Parallel tuned contactless power pickup using saturable core reactor," in 2010 IEEE International Conference on Sustainable Energy Technologies (ICSET), 2010, pp. 1-6.

[6] Y. Lim, H. Tang, S. Lim, and J. Park, "An adaptive impedance-matching network based on a novel capacitor matrix for wireless power transfer," IEEE Trans. Power Electron., vol. 29, no. 8, pp. 4403-4413, 2014.

[7] A. P. Hu and S. Hussmann, "Improved power flow control for contactless moving sensor applications," IEEE Power Electron. Lett., vol. 2, no. 4, pp. 135-138, 2004.

[8] M. Yang, X. Li, Z. Ao, and Y. Wang, "Transferred Power Control for ICPT Pick-ups Utilizing Dynamically Switched Inductor*," Energy Procedia, vol. 16, no. PART C, pp. 1440-1447, 2012.

[9] N. Keeling, G. A. Covic, F. Hao, L. George, and J. T. Boys, "Variable tuning in LCL compensated contactless power transfer pickups," in 2009 IEEE Energy Conversion Congress and Exposition, 2009, pp. 1826-1832.

[10] J.-U. W. Hsu, A. P. Hu, and A. Swain, “A Wireless Power Pickup Based on Directional Tuning Control of Magnetic Amplifier,” IEEE Trans. Ind. Electron., vol. 56, no. 7, pp. 2771-2781, Jul. 2009.

[11] Z. Pantic and S. M. Lukic, "Framework and Topology for Active Tuning of Parallel Compensated Receivers in Power Transfer Systems," IEEE Trans. Power Electron., vol. 27, no. 11, pp. 4503-4513, 2012.

[12] A. Saberkari, S. Ziabakhsh, H. Martinez, and E. Alarcón, "Active inductor-based tunable impedance matching network for RF power amplifier application,” Integr. VLSI J., Aug. 2015.

[13] S. Singer, “Loss-free gyrator realization,” IEEE Trans. Circuits Syst., vol. 35, no. 1, pp. 26-34, 1988.

[14] R. Y. Barazarte, G. G. Gonzalez, and M. Ehsani, "Generalized Gyrator Theory," IEEE Trans. Power Electron., vol. 25, no. 7, pp. 1832-1837, Jul. 2010.

[15] M. Saad, N. Egidos, E. Bou-Balust, and E. Alarcon, "On tunable switch-mode reactive networks: A gyratorbased resonator emulation," in Proceedings - IEEE International Symposium on Circuits and Systems, 2016, vol. 2016-July, pp. 642-645.

[16] M. Saad and E. Alarcon, "Tunable switch-mode emulated inductive elements for enhanced power converter miniaturization," in Industrial Electronics Society, IECON 2016 - 42nd Annual Conference of the IEEE, 2016, pp. 1184-1189.

[17] D. Shmilovitz, "Loss-free complex impedance network elements," IEEE Trans. Circuits Syst. I Regul. Pap., vol. 53, no. 3, pp. 704-711, Mar. 2006.

[18] E. Rodríguez Vilamitjana, A. El Aroudi, and E. Alarcón, Chaos in switching converters for power management : designing for prediction and control. New York, NY: Springer, 2013. 